\title{
Pathophysiology of Anemia in HIV-Infected Children Exposed to Malaria
}

\author{
Cinta Moraleda, ${ }^{1,2 \star}$ Ruth Aguilar, ${ }^{1,2,3}$ Llorenç Quintó, ${ }^{1}$ Tacilta Nhampossa, ${ }^{2}$ Montserrat Renom, ${ }^{1,2}$ Augusto Nhabomba, ${ }^{2}$ \\ María Ruperez, ${ }^{1,2}$ John J. Aponte, ${ }^{1,2}$ Ariel H. Achtman, ${ }^{4,5}$ María del Mar Mañú Pereira, ${ }^{6}$ Louis Schofield, ${ }^{4,5,7}$ Pedro L. Alonso, ${ }^{1,2}$ \\ Eusebio Macete, ${ }^{2,8}$ and Clara Menéndez $z^{1,2,3}$ \\ ${ }^{1}$ ISGlobal, Hospital Clínic - Universitat de Barcelona, Barcelona, Spain; ${ }^{2}$ Manhiça Health Research Center (CISM), Manhiça, Mozambique; ${ }^{3}$ CIBER \\ Epidemiology and Public Health (CIBERESP), Barcelona, Spain; ${ }^{4}$ Walter and Eliza Hall Institute for Medical Research, Victoria, Australia; \\ ${ }^{5}$ Department of Medical Biology, The University of Melbourne, Victoria, Australia; ${ }^{6}$ Red Blood Cell Pathology-Centre for Rare Diseases, Vall \\ d'Hebron Research Institute, University Hospital Vall d'Hebron, Barcelona, Spain; ${ }^{7}$ Australian Institute of Tropical Health and Medicine, James \\ Cook University, Douglas, Australia; ${ }^{8}$ National Directorate of Health, Ministry of Health, Maputo, Mozambique
}

\begin{abstract}
Anemia is a common condition in HIV-infected children; however, its pathophysiology and the contribution of frequent causes of anemia such as iron deficiency (ID) and malaria are poorly understood. We carried out an ancillary study on the effect of HIV on anemia as part of a case-control study on risk factors of anemia among Mozambican children aged 1-59 months with documented HIV status. Of them, 390 children were admitted to the hospital with anemia (hemoglobin $[\mathrm{Hb}]<11 \mathrm{~g} / \mathrm{dL}$ ), whereas 272 children without anemia $(\mathrm{Hb} \geq 11 \mathrm{~g} / \mathrm{dL}$ ) were recruited in the community. We assessed differences by HIV status in the presentation of anemia etiological factors and the effect of HIV infection on the association of each factor with anemia. Among the 99 HIV-infected and 563 uninfected children included, HIV-infected anemic children had an increased risk of undernutrition $(P<0.0001)$, Epstein-Barr virus infection $(P<0.0001)$, bacteremia $(P=0.0060)$, a decreased risk of malaria $(P<0.0001)$, and a similar risk of $\operatorname{ID}(P=0.7371)$ compared with anemic-uninfected children. HIV-infected children were significantly less likely to have anemia associated with Plasmodium falciparum hyperparasitemia $(P=0.0444)$ and had a lower prevalence of parasitemia in the bone marrow $(\mathrm{BM})(P<0.0001)$ than anemic-uninfected children. Levels of BM erythropoiesis and dyserythropoiesis were comparable between groups. These findings suggest that the pathophysiology of anemia among HIV-infected malaria-exposed children is not related to HIV-specific effects. For unclear reasons, HIV-infected children had reduced risk of malaria infection, whereas ID prevalence was comparable in HIV-infected and uninfected children, suggesting that iron supplementation recommendations should not be different in HIV-infected children.
\end{abstract}

\section{INTRODUCTION}

Anemia is a frequent complication of HIV infection, associated with disease progression. ${ }^{1}$ Both anemia and HIV infection are frequent in sub-Saharan Africa, where other infections and malnutrition are also prevalent. ${ }^{1}$ In high-infectious pressure settings, the diagnosis of anemia and its management is hampered because of the multifactorial etiology of anemia. ${ }^{2}$ The pathophysiology of HIV-associated anemia in malariaendemic areas is not entirely understood, especially in children. ${ }^{1}$ Studies among HIV-infected children using bone marrow (BM) samples are scarce because of the lack of expertise, especially in low-income settings in carrying out BM examination, which is the gold standard to assess the iron status and the only accurate way to analyze it. ${ }^{2}$ However, most studies using BM examination are retrospective or case reports of small size with no control group, which prevents having accurate information on the main contributors to anemia in HIV-infected children. ${ }^{1,3-5}$ For instance, it is unclear how much frequent causes of anemia, such as iron deficiency (ID) and malaria, contribute to anemia of HIV-infected children. Similarly, data on whether HIV infection modifies the risk of anemia associated with some etiologic factors like ID or malnutrition are limited. ${ }^{1,6,7}$

Therapeutic alternatives to manage anemia among HIVinfected patients are limited. Subcutaneous erythropoietin (EPO) is not easily accessible and affordable in low-income countries. In addition, iron supplementation to HIV-infected individuals remains controversial because of the lack of

*Address correspondence to Cinta Moraleda, ISGlobal, Hospital Clínic - Universitat de Barcelona, Rosselló 132, 5th floor, Barcelona E-08036, Spain. E-mail: cinta.moraleda@isglobal.org evidence on the contribution of ID to HIV-related anemia and the fear of harmful effects of iron supplementation in malariaendemic areas. ${ }^{1,8}$ As a consequence, most HIV-infected children in low-income countries present some degree of anemia.

As part of a case-control study on the etiology of anemia among Mozambican children aged 1-59 months, we conducted a secondary analysis with the goal of contributing to improving the management and prevention of anemia in HIVinfected children and the objectives of 1) assessing the association between HIV status and anemia-related variables among anemic children, 2) describing the pathophysiology of anemia in HIV-infected children, and 3) analyzing the effect modifier of HIV status on the risk of anemia associated with frequent etiologic factors of anemia.

\section{MATERIALS AND METHODS}

Study site. The study was carried out at the Manhiça District Hospital (MDH) and the Centro de Investigação em Saúde de Manhiça (CISM), Maputo Province, in southern Mozambique between October 2008 and August 2010. Since 1996, the CISM runs a continuous demographic surveillance system (DSS) that covered 130,351 inhabitants at the time of the study. The CISM study area has been described in detail elsewhere. ${ }^{9}$ During the study, HIV prevalence in adults (18-47 years of age) and children ( $\leq 11$ years old) was $38 \%$ and $1 \%$, respectively. ${ }^{10}$ The risk of mother-to-child HIV transmission in the first month of life and at 1 year of age was $9 \%$ and $27 \%$, respectively. ${ }^{11}$ Most HIV-infected children had advanced disease at the time of diagnosis. ${ }^{12}$ Clinical management of HIV-infected children followed WHO guidelines at the time, namely, starting antiretroviral treatment (ART) in infancy and 
following the WHO criteria for children aged $>1$ year. ${ }^{13}$ Malaria transmission in the area, mainly due to Plasmodium falciparum $(P f)$, was perennial, with some seasonality. Community-based prevalence of anemia (hemoglobin $[\mathrm{Hb}]<11 \mathrm{~g} / \mathrm{dL}$ ) in children younger than 5 years in Manhiça was $91 \%$ (C. Moraleda, personal communication). G6PD deficiency prevalence was around 9\%, $\alpha$-thalassemia (a-/a-) was about 15\%, and sickle cell disease and $\beta$-thalassemia were both less than $1 \%{ }^{14}$

Study participants and procedures. This was an ancillary study within a case-control study on etiologic factors of anemia in children aged 1-59 months. ${ }^{14}$ In brief, cases were children attending the MDH emergency department between October 2008 and August 2010 with anemia defined as an $\mathrm{Hb}$ concentration $<11 \mathrm{~g} / \mathrm{dL}$ in capillary samples, ${ }^{15}$ determined by HemoCue ${ }^{\circledR}$ HB $201^{+}$system (Änghelom, Sweden), and without a history of blood transfusion in the preceding 4 weeks. Controls were children of the same age randomly selected from the DSS who were visited at the community by study personnel. Their $\mathrm{Hb}$ level was measured at home, and those children with an $\mathrm{Hb}$ level $\geq 11 \mathrm{~g} / \mathrm{dL}$ were invited to attend the $\mathrm{MDH}$ for a study visit. At study entry, a standardized questionnaire on clinical and demographic information was administered to cases and controls, and capillary and venous blood samples were collected. Urine and stool samples were also collected when provided. Biological samples were processed at the CISM laboratory. Some samples were shipped to Barcelona, Spain, for further analysis. Bone marrow aspiration was performed under conscious sedation and only in anemic children (cases) $\geq 3$ months of age without any medical contraindication or any risk at the discretion of the responsible clinician. Study children were offered voluntary HIV counseling and testing. Only children with available HIV-positive or negative test results or with previously documented HIV status were included in this analysis (see Figure 1). During the conduct of the study and following national guidelines, HIV status was assessed using the Determine HIV-1/2 Rapid Test (Abbott Laboratories, Abbott Park, IL), and positive results were confirmed by the Uni-Gold Rapid Test (Trinity Biotech Co., Wicklow, Ireland). HIV infection was confirmed using the HIV-1 DNA-PCR kit (Roche Molecular Systems, Branchburg, $\mathrm{NJ}$ ) in children with discordant results and in those aged $<18$ months who were positive by both HIV rapid tests. All HIVinfected children whose HIV status was unknown before recruitment were further assessed at the HIV outpatient clinic. Study definitions are summarized in Supplemental Table S1.

Laboratory methods. Blood counts other than $\mathrm{Hb}$ measurements were performed using a Sysmex analyzer KX21N and later an XT-2000i (Sysmex Long Grove, IL). Reticulocyte counts were estimated by microscopy on cresyl blue-stained blood smears. Plasmodium falciparum infection was identified by microscopy in thick and thin Giemsa-stained blood films. Plasmodium falciparum-specific real-time quantitative PCR (qPCR) was performed on microscopically negative samples at the Hospital Clinic of Barcelona, Spain. Blood cultures were performed on an automated system (BACTEC ${ }^{\circledR}$ 9050; BectonDickinson, Franklin Lake, NJ). Epstein-Barr virus (EBV) and parvovirus B19 (PV-B19) were detected by qPCR using the Artus EBV RG PCR and the Artus Parvo B19 RG PCR kits (QIAGEN), respectively. Epstein-Barr virus serology was performed using the kits Merifluor ${ }^{\circledR}$ EBV anti-antigen capsid virus (VCA) IgM IFA (Meridian Bioscience, Cincinnati, $O H$ ) and CaptiaTM EBV VCA IgG (Trinity Biotech, Wicklow, Ireland). Epstein-Barr virus tests were performed at the Hospital Clínic, Barcelona, Spain. Stool parasites were examined by microscopy using the merthiolate-iodine-formalin concentrations method. ${ }^{16}$ Albumin, prealbumin, and C-reactive protein (CRP) were measured in plasma using an ADVIA 2400 analyzer (Siemens Healthcare, Spain), and folic acid, vitamin B12, and

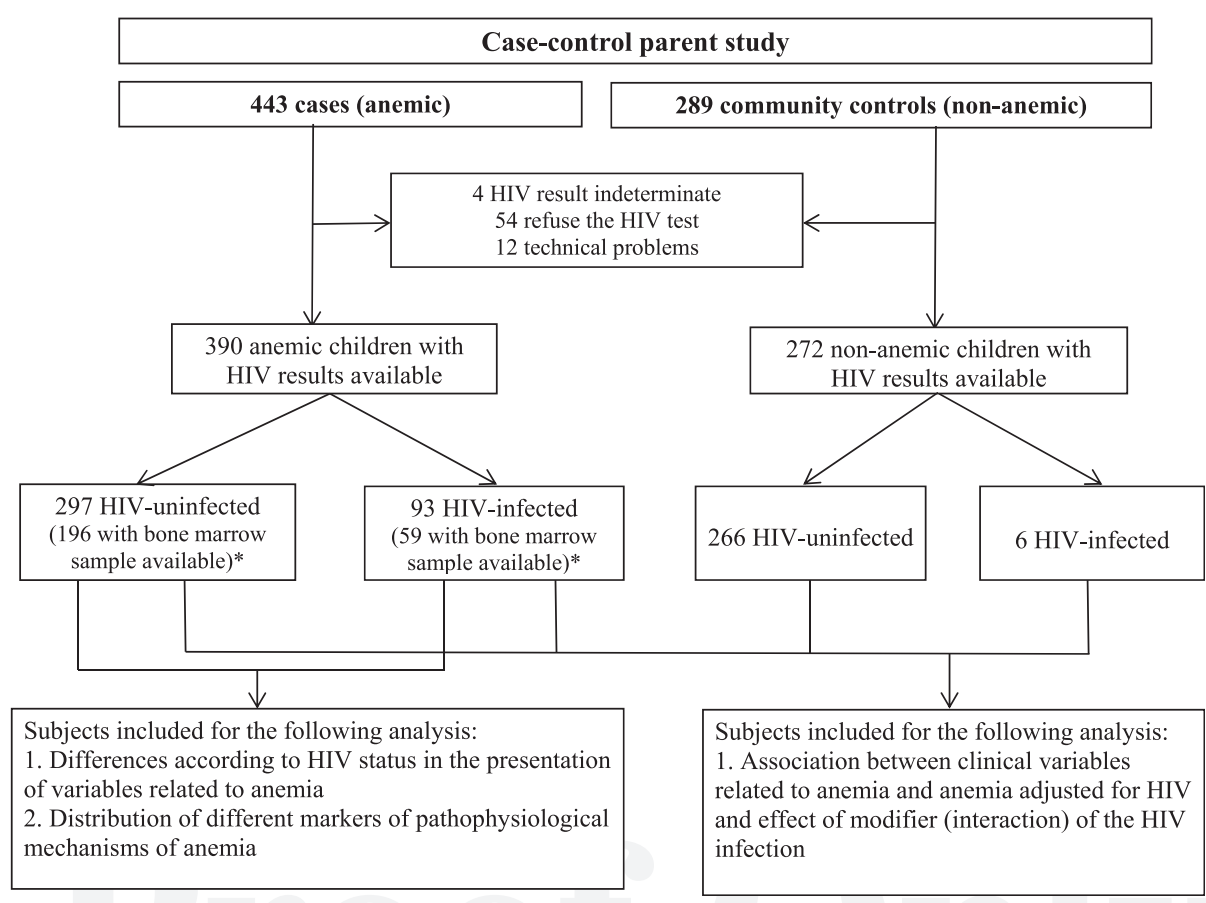

FIGURE 1. Study flowchart. *It was not possible to perform all the procedures in each bone marrow (BW) samples because of low quantity and/or technical problems. The number of samples with the different results available was included in the corresponding table. 
ferritin were determined with an ADVIA Centaur (Siemens Healthcare). Plasma-soluble transferrin receptors were determined using a BN-II nephelometer (Dade-Siemens Healthcare), vitamin A was determined by reversed-phase high-performance liquid chromatography, and EPO was quantified using the Quantikine human EPO immune-analysis kit (R\&D Systems). Bone marrow smears were stained by the Perls' Prussian blue method, and the BM iron content was measured semi-quantitatively by the amount of blue-stained hemosiderin pearls. Asexual parasitemia in BM was estimated as the percentage of infected red blood cells (iRBCs) in 1,000 RBCs, and gametocytes were counted in 100 fields. Hemozoin content was assessed in BM macroscopic cell aggregates, which were composed of stromal and parenchymal cells and were needed for an optimal BM smear examination. Hemozoin was detected by light microscopy as coarse brown granular material with birefringence under polarized light. ${ }^{17}$ Erythropoiesis was assessed by three methods, namely, morphological quantification, BM flow cytometric analysis, and transcriptional score (Supplemental Table S1) (Achtman et al., manuscript in preparation). Biochemistry and BM tests were analyzed in the Hospital Clinic in Spain, whereas erythropoiesis analysis was performed at the Walter and Eliza Hall Institute for Medical Research, Victoria, Australia.

Statistical analysis. Data were double entered using Microsoft Visual FoxPro 5.0 (Microsoft Corp.), and analyses were performed using Stata/SE 14.0 for Mac (64-bit Intel) (StataCorp. 2015: Release 14. College Station, TX: StataCorp LP). Differences by HIV status in the presentation of anemia etiological factors were assessed only in the group of anemic children by logistic regression with Firth's penalized likelihood, except for the reticulocyte production index and BM measures of erythropoiesis, which were analyzed by linear regression (flow cytometry in log scale). Logistic regression models with Firth's penalized likelihood and 95\% Cls based on the Profile penalized log likelihood were estimated to assess the impact of HIV on the association of each potential etiological factor with anemia using data from anemic and non-anemic children. ${ }^{18}$ Factor-specific multivariable models were estimated using the factor, HIV, and its interaction as independent variables. The modification of the factor-specific association with anemia (interaction/effect modifier) was assessed by the odds ratio (OR) corresponding to the interaction coefficient, that is, the ratio and $95 \% \mathrm{Cl}$ of the OR (ROR) among HIV-infected children over the OR among HIVnegative children.

Fractional polynomial analysis was used to compare the relationship among plasma EPO and $\mathrm{Hb}$ concentrations among the HIV-infected and uninfected children using the estimated equation:

$$
\begin{aligned}
\ln (\mathrm{EPO})= & -5.91 \times \ln (\mathrm{Hb} / 10)+3.02 \\
& \times \sqrt{\mathrm{Hb} / 10-0.41 \times \text { HIVstatus }+0.06 \times \text { HIVstatus } \times \mathrm{Hb}}
\end{aligned}
$$

where "HIVstatus" is an indicator variable that equals 0 or 1 for HIV-uninfected or HIV-infected children, respectively.

Ethics. The protocol of the case-control study was approved by the National Mozambican Ethics Committee and the Hospital Clínic of Barcelona Ethics Review Committee. The parents/guardians of all children included in the study provided written informed consent after being informed of the goals, procedures, benefits, and risks of taking a BM sample from their child. No financial or material inducement for participation was offered. Voluntary counseling and testing for HIV were provided to all study participants at the enrolment of the case-control study. The child was tested for HIV only after consent was obtained. Permission for further analyses related to the study was included in the informed consent form.

\section{RESULTS}

Study population. Demographic and clinical features of study children are shown in Table 1. In the overarching case-control study, 443 anemic children were recruited as cases and 289 non-anemic children as community controls. Of them, $662(90 \%)$ children had an HIV result and were included in this analysis, with 563 being (85\%) HIV uninfected (297 [53\%] cases and 266 [47\%] controls) and 99 (15\%) HIV infected (93 [94\%] cases and six [6\%] controls). Four (1\%) children had an indeterminate HIV test result; in 54 (7\%) children, the parents refused to perform the HIV test, and in 12 (2\%) children, the sample was not adequate (see Figure 1).

HIV status and anemia-related variables among anemic children. Association between HIV infection and anemiarelated variables among anemic children is summarized in Table 2. The risks of having a positive EBV viral load and EBV reactivation, bacteremia, albumin deficiency, and moderate and severe malnutrition (see definitions in Supplemental Table S1) were statistically significantly higher among anemic HIVinfected children than among anemic uninfected children. On the other hand, the risks of having Pf infection and clinical malaria were lower among anemic HIV-infected than uninfected anemic children (Table 2). HIV-infected anemic children also had a lower prevalence of $P f$ parasitemia, gametocytes, and hemozoin in BM than anemic uninfected children. However, the prevalence of malaria gametocytes or hemozoin in BM among children without $P f$ in peripheral blood was similar in HIVinfected and uninfected children (Table 2). No statistically significant associations between having HIV infection and iron, vitamin A or vitamin B12 deficiencies, or intestinal parasitic infections were found (Table 2). Among HIV-infected patients, two children had parvovirus infection; in both of them, RBC precursors were diminished in the BM without showing the characteristic gigantic proerythroblasts.

HIV status and markers of anemia pathophysiology. The association of HIV infection with markers of the main mechanisms of anemia pathophysiology, namely, blood loss, RBC destruction, and changes in erythropoiesis, were investigated. Among anemic children, those with HIV infection had fewer erythroid precursors by flow cytometry than uninfected children (1.6\% versus $2.4 \%,[P=0.0227])$, but these differences were not statistically significant after adjusting for $P f$ infection $(P=0.2748)$ (Table 3$)$. Hemolysis was less frequent among HIV-infected children than among uninfected children (6/65 [9\%] versus $7 / 246$ [29\%], $P=0.0374)$. The frequency of dyserythropoiesis was similar between HIV-infected and uninfected children (27/46 [59\%] versus 103/144 [72\%], $P=$ 0.3923 ) (Table 3). The proportion of participants with elevated EPO was also similar among HIV-infected and uninfected children in the unadjusted analysis ( $P=0.3418$ ), but after adjusting for $P f$ infection, the proportion was significantly higher in HIV-infected children than uninfected children (69/88 [78\%] versus 215/294 [73\%]; $P=0.0434$ ) (Table 3). Nevertheless, in the regression analysis, EPO synthesis in response to anemia was not significantly different among the two 
TABLE 1

Demographic and clinical characteristics of the study participants

\begin{tabular}{|c|c|c|c|c|c|}
\hline \multirow{2}{*}{ Variable } & \multicolumn{3}{|c|}{ Study group } & \multirow[b]{2}{*}{ Total $^{*}$} & \multirow[b]{2}{*}{$P$-value } \\
\hline & Non-anemi & & Anemic & & \\
\hline HIV-positive, $n$ (\%) & & & $93(94)$ & $99(100)$ & \\
\hline & & & & & \\
\hline Age (months) $\dagger$ & $17.83(18.23)[6]$ & & 19.90 (14.78) [93] & 19.78 (14.90) [99] & $0.7435 \ddagger$ \\
\hline Gender,§ $\boldsymbol{n}$ (\%) & & & & & \\
\hline Male & $2(33)$ & & $53(57)$ & $55(56)$ & $0.4020^{\|}$ \\
\hline Female & $4(67)$ & & $40(43)$ & $44(44)$ & \\
\hline Total & $6(100)$ & & $93(100)$ & $99(100)$ & \\
\hline Fever§ & $0 / 6(0 \%)$ & & 80/93 (86\%) & $80 / 99(81 \%)$ & $<0.0001^{\|}$ \\
\hline Hemoglobin $†$ & $11.40(0.17)[6]$ & & 7.55 (1.94) [93] & $7.79(2.10)[99]$ & $<0.0001 \ddagger$ \\
\hline Hemoglobin $<7 \mathrm{~g} / \mathrm{dL} \S$ & $0 / 6(0 \%)$ & & $33 / 93(35 \%)$ & 33/99 (33) & $0.1744^{\|}$ \\
\hline $\mathrm{CRP}(\mathrm{mg} / \mathrm{dL}) \dagger$ & $0.21(0.27)[5]$ & & $8.99(8.64)[87]$ & $8.52(8.63)[92]$ & $0.0262 \ddagger$ \\
\hline $\begin{array}{l}\text { HIV-infected status known before } \\
\text { study§ }\end{array}$ & $1 / 6(17 \%)$ & & $43 / 93(46 \%)$ & 44/99 (44) & $0.2221^{\|}$ \\
\hline Cotrimoxazole prophylaxis,§n (\%) & $\begin{array}{l}\text { No } \\
\text { Yes } \\
\text { NA }\end{array}$ & $\begin{array}{l}0(0) \\
0(0) \\
6(100)\end{array}$ & $\begin{array}{l}27(29) \\
11(12) \\
55(59)\end{array}$ & $\begin{array}{l}27(27) \\
11(11) \\
61(62)\end{array}$ & $0.2260^{\|}$ \\
\hline $\begin{array}{l}\text { Highly active antiretroviral therapy } \\
\text { treatment, } \S \boldsymbol{n}(\%)\end{array}$ & $\begin{array}{l}\text { No } \\
\text { Yes } \\
\text { NA }\end{array}$ & $\begin{array}{l}0(0) \\
0(0) \\
6(100)\end{array}$ & $\begin{array}{r}70(75) \\
9(10) \\
14(15)\end{array}$ & $\begin{array}{c}70(71) \\
9(9) \\
20(20)\end{array}$ & - \\
\hline HIV viral load copies $/ \mathrm{mL}, \S \boldsymbol{n}(\%)$ & $\begin{array}{c}<109 \\
\geq 10 \uparrow \\
\text { Undetectable } \\
\text { NA }\end{array}$ & $\begin{array}{l}0(0) \\
1(17) \\
2(33) \\
3(50)\end{array}$ & $\begin{array}{c}14(15) \\
64(69) \\
12(13) \\
3(3)\end{array}$ & $\begin{array}{c}14(14) \\
65(66) \\
14(14) \\
6(6)\end{array}$ & $0.0009^{\|}$ \\
\hline HIV-negative, $n$ (\%) & $266(47)$ & & $297(53)$ & $563(100)$ & \\
\hline Age (months) $\dagger$ & 27.37 (18.62) [266 & & 19.45 (13.91) [297] & 23.19 (16.76) [563] & $<0.0001 \ddagger$ \\
\hline $\begin{array}{l}\text { Gender,§ } \boldsymbol{n}(\%) \\
\quad \text { Male }\end{array}$ & $121(45)$ & & $173(58)$ & $294(52)$ & 0.0025 \\
\hline Female & $145(55)$ & & $124(42)$ & $269(48)$ & \\
\hline Total & $266(100)$ & & $297(100)$ & $563(100)$ & \\
\hline Fever§ & $1 / 266(0 \%)$ & & $262 / 297(88 \%)$ & $263 / 563(47 \%)$ & $<0.00019$ \\
\hline Hemoglobin† & $11.67(0.76)$ [266] & & $8.02(1.96)[297]$ & $9.74(2.37)[563]$ & $<0.0001 \ddagger$ \\
\hline Hemoglobin $<7 \mathrm{~g} / \mathrm{dL} \S$ & $0 / 266(0 \%)$ & & $84 / 297(28 \%)$ & $84 / 563(15 \%)$ & $<0.00019$ \\
\hline $\mathrm{CRP}(\mathrm{mg} / \mathrm{dL}) \dagger$ & $0.38(0.95)[262]$ & & $8.34(7.75)$ [293] & $4.58(6.92)[555]$ & $<0.0001 \mp$ \\
\hline
\end{tabular}

$\mathrm{CRP}=\mathrm{C}$-reactive protein; NA $=$ not available (missing information). Bold figures: statistically significant results.

${ }^{\star}$ Because it was not possible to obtain the need amount of blood in all the children, several analyses were not performed in some of them, so the denominator was not always as the total $N$. †Arithmetic mean (SD) (n).

$\ddagger t$-test.

$\S n$ (column percentage).

$\|$ Fisher's exact test.

$\uparrow$ Chi-squared test.

groups of children. No differences were observed either for HIV status $(P=0.3236)$ or HIV infection with $\mathrm{Hb}$ level interaction $(P=0.2424)$ (Figure 2).

Effect of HIV infection on the etiology of anemia. The effect modifier of HIV infection on the factors related to anemia is shown in Table 4. We did not observe an interaction of HIV with the likelihood of anemia in the presence of positive EBV viral load, bacteremia, albumin deficiency, and malnutrition. The distribution of studied variables and the presence of anemia by HIV groups are shown in Supplemental Table S2.
The association of hyperparasitemic $P f$ infection with anemia among HIV-infected children was 67.5 times lower (1/effect of modification) than that among uninfected children (ROR: 0.02; $95 \% \mathrm{Cl}: 0.00,0.90 ; \mathrm{P}=0.0444)$. Thus, for every case of anemia among HIV-infected children with hyperparasitemic Pf infection, 68 cases of hyperparasitemic $P f$ infection among anemic HIV-uninfected children would be expected.

The association among ID (assessed by the ratio of soluble transferrin receptor to log ferritin [TfR-F] index) and anemia among HIV-infected children was 15.8 times lower 
TABLE 2

Differences according to HIV status in the presentation of variables related to anemia among anemic children

\begin{tabular}{|c|c|c|c|c|}
\hline \multirow[b]{2}{*}{ Variable } & \multicolumn{2}{|c|}{$\mathrm{HIV}^{*}$} & \multirow[b]{2}{*}{ Odds ratio $(95 \% \mathrm{Cl})$} & \multirow[b]{2}{*}{$P$-value } \\
\hline & Negative & Positive & & \\
\hline ID by BM† & 101/127 (80\%) & $31 / 40(78 \%)$ & $0.87(0.37,2.01)$ & $0.7371 \neq$ \\
\hline ID by TfR-F index by C-reactive protein* & $184 / 262(70 \%)$ & $47 / 72(65 \%)$ & $0.79(0.46,1.37)$ & $0.4064 \dagger$ \\
\hline Folate deficiency ${ }^{*}$ & $0 / 261(0 \%)$ & $0 / 72(0 \%)$ & $3.61(0.07,183.35)$ & $0.5222 \dagger$ \\
\hline Vitamin A deficiency* & $205 / 294$ (70\%) & $59 / 88(67 \%)$ & $0.88(0.53,1.46)$ & $0.6159 \dagger$ \\
\hline Vitamin B12 deficiency ${ }^{*}$ & $50 / 283(18 \%)$ & $11 / 80(14 \%)$ & $0.77(0.38,1.53)$ & $0.4496 \dagger$ \\
\hline Albumin deficiency* & $104 / 293(35 \%)$ & $53 / 87(61 \%)$ & $2.81(1.72,4.59)$ & $<0.0001 \dagger$ \\
\hline Prealbumin deficiency* & 265/292 (91\%) & $77 / 86(90 \%)$ & $0.84(0.39,1.84)$ & $0.6720 \dagger$ \\
\hline Wasting $(\mathrm{WHZ}<-2 \mathrm{SD})^{\star}$ & $66 / 293(23 \%)$ & $56 / 93(60 \%)$ & $5.15(3.14,8.46)$ & $<0.0001 \dagger$ \\
\hline Stunting $(\mathrm{HAZ}<-2 \mathrm{SD})^{*}$ & $77 / 293$ (26\%) & $52 / 93(56 \%)$ & $3.53(2.18,5.73)$ & $<0.0001 \dagger$ \\
\hline Underweight $(W A Z<-2 S D)^{\star}$ & $114 / 297(38 \%)$ & $67 / 93(72 \%)$ & $4.08(2.46,6.77)$ & $<0.0001 \dagger$ \\
\hline Severe wasting $(\mathrm{WHZ}<-3 \mathrm{SD})^{*}$ & 28/293 (10\%) & $39 / 93(42 \%)$ & $6.75(3.85,11.86)$ & $<0.0001 \dagger$ \\
\hline Severe stunting $(H A Z<-3 S D)^{\star}$ & $19 / 293(6 \%)$ & $35 / 93(38 \%)$ & $8.54(4.59,15.89)$ & $<0.0001 \dagger$ \\
\hline Severe underweight $(\mathrm{WAZ}<-3 \mathrm{SD})^{*}$ & $56 / 297(19 \%)$ & $53 / 93(57 \%)$ & $5.65(3.42,9.31)$ & $<0.0001 \dagger$ \\
\hline Parvovirus B19 infection* & 23/297 (8\%) & 2/93 (2\%) & $0.32(0.08,1.20)$ & $0.0912 \dagger$ \\
\hline EBV viral load positive* & $61 / 296(21 \%)$ & $42 / 93(45 \%)$ & $3.16(1.93,5.18)$ & $<0.0001 \dagger$ \\
\hline Acute EBV infection* & $14 / 284(5 \%)$ & $8 / 85(9 \%)$ & $2.05(0.85,4.95)$ & $0.1123 \dagger$ \\
\hline EBV reactivation* ${ }^{\star}$ & $35 / 284(12 \%)$ & $26 / 85(31 \%)$ & $3.13(1.76,5.57)$ & $0.0001 \dagger$ \\
\hline Bacteremia* & $17 / 287(6 \%)$ & 14/93 (15\%) & $2.82(1.35,5.91)$ & $0.0060 \dagger$ \\
\hline Clinical malaria & $131 / 286(46 \%)$ & $18 / 90(20 \%)$ & $0.30(0.17,0.53)$ & $<0.0001 \dagger$ \\
\hline$P f$ infection* ${ }^{*}$ & $137 / 286(48 \%)$ & 19/90 (21\%) & $0.30(0.17,0.51)$ & $<0.0001 \dagger$ \\
\hline Hyperparasitemic $P f^{*}$ & $27 / 286(9 \%)$ & $5 / 90(6 \%)$ & $0.61(0.24,1.57)$ & $0.3018+$ \\
\hline Submicroscopic $P f^{\star}$ & $25 / 204(12 \%)$ & $10 / 83(12 \%)$ & $1.01(0.47,2.17)$ & $0.9886 \dagger$ \\
\hline Pf parasites in $\mathrm{BM}^{*}$ & $90 / 182(49 \%)$ & $10 / 58(17 \%)$ & $0.22(0.11,0.46)$ & $<0.0001 \dagger$ \\
\hline Hemozoin in $\mathrm{BM}^{\star}$ & $80 / 144(56 \%)$ & 9/44 (20\%) & $0.21(0.10,0.47)$ & $0.0001 \dagger$ \\
\hline Gametocytes BM* & $54 / 182(30 \%)$ & $6 / 57(11 \%)$ & $0.30(0.12,0.71)$ & $0.0066+$ \\
\hline Hemozoin in $\mathrm{BM}$ without $P f \mathrm{Pl}^{*}$ & $12 / 135(9 \%)$ & $3 / 43(7 \%)$ & $0.85(0.25,2.94)$ & $0.8023 \dagger$ \\
\hline$P f$ parasites in $\mathrm{BM}$ without $P f \mathrm{PI}^{\star}$ & $3 / 173(2 \%)$ & $1 / 56(2 \%)$ & $1.32(0.19,9.13)$ & $0.7807 \dagger$ \\
\hline Hookworm infection* & $4 / 133(3 \%)$ & $1 / 51(2 \%)$ & $0.85(0.13,5.58)$ & $0.8698 \dagger$ \\
\hline
\end{tabular}

(1/interaction) than that among uninfected children. This difference is borderline significant at the 0.05 level (ROR: 0.063; 95\% Cl: 0.003, 1.248; $P$-value: 0.0696$)$. The latter indicates that for every case of anemia among HIV-infected children with ID, 16 cases of anemia among uninfected children with ID would be expected. By contrast, among HIV-infected children, the odds of anemia in those with ID (by BM iron content) were nonsignificantly lower than those among those without ID (OR: 0.21; 95\% Cl: 0.01, 4.00; P = 0.2971).

\section{DISCUSSION}

To our knowledge, this is the widest study on the pathophysiology of anemia in HIV-infected children exposed to

TABLE 3

Distribution of different markers of pathophysiological mechanisms of anemia among anemic children according to HIV status

\begin{tabular}{|c|c|c|c|c|}
\hline Pathophysiological marker & HIV-negative* & HIV-positive $^{\star}$ & $P$-value & $P$-value (adjusted for Plasmodium falciparum. infection) \\
\hline \multicolumn{5}{|l|}{ Blood loss } \\
\hline Blood loss in feces $\dagger$ & $3 / 133(2 \%)$ & $3 / 51(6 \%)$ & $0.2012 \ddagger$ & $0.2198 \ddagger$ \\
\hline Blood loss in urine† & 1/297 (0\%) & $0 / 93(0 \%)$ & $0.9730 \ddagger$ & $0.7434 \ddagger$ \\
\hline Hemolysis & $71 / 246(29 \%)$ & 6/65 (9\%) & $0.0026 \ddagger$ & $0.0374 \ddagger$ \\
\hline Erythropoietin response $^{a}(>20 \mathrm{U} / \mathrm{L}) \dagger$ & $215 / 294(73 \%)$ & $69 / 88(78 \%)$ & $0.3418 \ddagger$ & $0.0434 \ddagger$ \\
\hline \multicolumn{5}{|l|}{ Red cell production failure } \\
\hline Dyserythropoiesis $†$ & 103/144 (72\%) & $27 / 46(59 \%)$ & $0.1018 \ddagger$ & $0.3923 \ddagger$ \\
\hline Reticulocyte Production index $<2 \dagger$ & $204 / 215$ (95\%) & $66 / 69(96 \%)$ & $0.9158 \ddagger$ & $0.5657 \ddagger$ \\
\hline \% Erythroid cells ${ }^{\mathrm{b}}$ (Morphology)§ $(n)$ & $29.71(16.63)[150]$ & $25.33(13.30)[51]$ & $0.0905^{\|}$ & 0.81659 \\
\hline$\%$ Erythroid cells ${ }^{\mathrm{C}}$ (Flow Cytometry)\# $(n)$ & $2.35(2.21)[147]$ & $1.64(1.57)[49]$ & $0.0227^{\|}$ & 0.2748 \\
\hline Transcriptional score of erythropoiesis $\S(n)$ & $0.06(0.25)[82]$ & $0.06(0.20)[18]$ & $0.9611^{\|}$ & 0.65899 \\
\hline
\end{tabular}

Bold figures: statistically significant results.

${ }^{\star}$ Because it was not possible to obtain the need amount of blood in all the children, several analyses were not performed in some of them, so the denominator was not always as the total $N$. a: Laboratory reference; $b$ : No of cells $/ 100$ nucleated cells; c: \% gated cells.

$\dagger n$ (column percentage).

$\ddagger$ Logistic regression with Firth's penalized likelihood.

$\S$ Arithmetic mean (SD) $[n]$

\|S Student's $t$-test.

ฯ Linear regression.

\# Geometric mean (SD) $[n]$. 


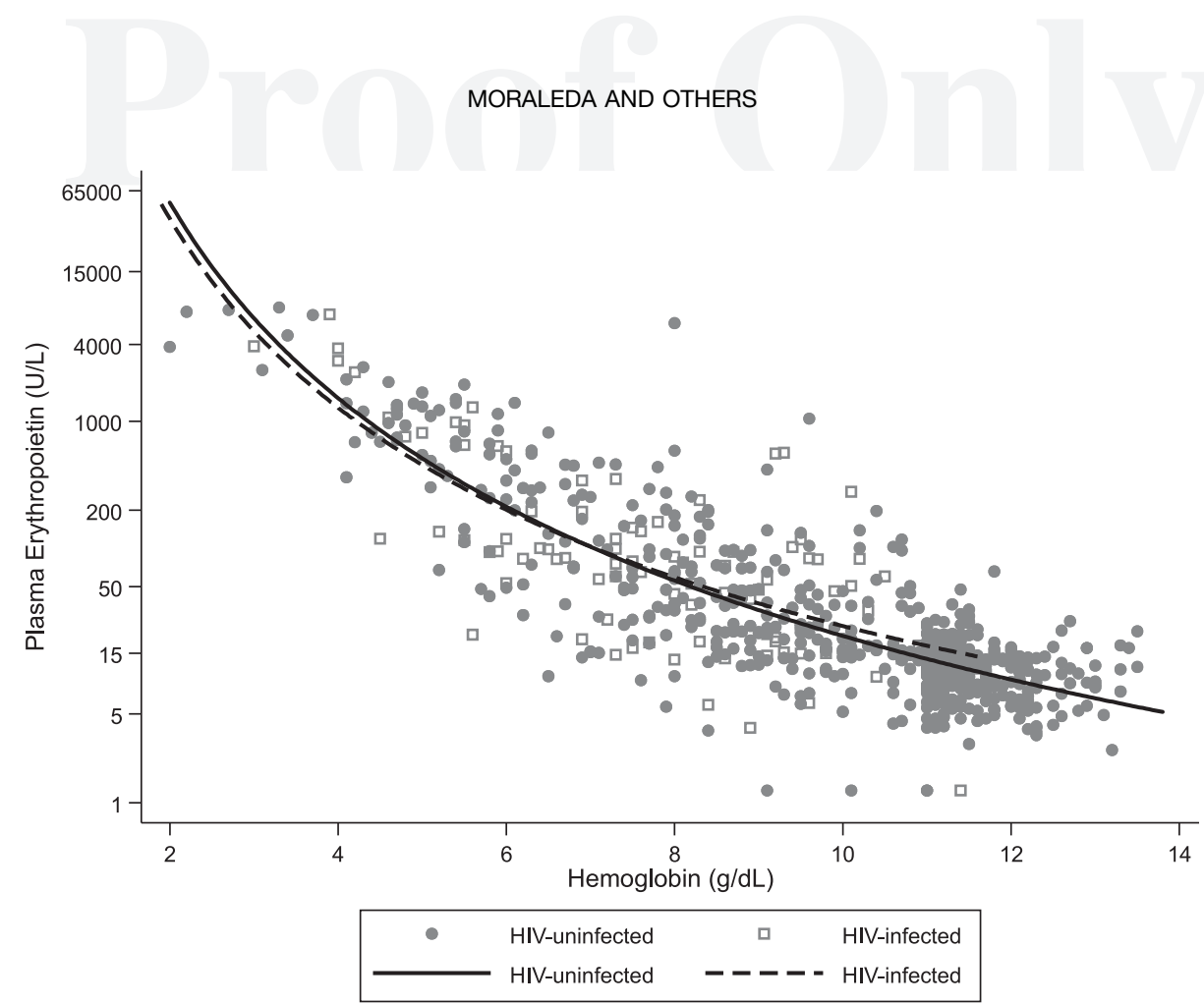

FIGURE 2. Erythropoietin levels in response to hemoglobin concentrations in HIV-infected and HIV-uninfected children.

malaria with BM examination. The findings show that BM function, as indicated by the number of erythroid precursors, was not different between anemic HIV-infected children and anemic uninfected children. Similarly, the prevalence of ID was not different among those groups. These observations indicate that these two mechanisms of anemia-BM dysfunction and ID_-are not playing a different role in the pathophysiology of anemia in HIV-infected children compared with uninfected children. Nevertheless, anemic HIV-infected children had an increased risk of malnutrition, positive EBV viral load and bacteremia, and a decreased risk of $P f$ infection. However, anemia associated with these conditions was not modified by HIV infection. Moreover, HIV-infected children had a lower risk of having anemia related to hyperparasitemia than uninfected children.

The pathogenesis of anemia in HIV-infected children is not completely understood. Previous studies reported that inflammation and the direct virus effect on the BM are the main pathophysiologic mechanisms of anemia. ${ }^{19}$ Bone marrow abnormalities are frequently observed in HIV-infected patients; however, these features are nonspecific and often associated with opportunistic infections. ${ }^{20}$ Our findings do not support previous reports suggesting that red cell production failure is an essential cause of anemia in HIV-infected children as compared with anemic uninfected children. ${ }^{20}$ In this study, the percentage of the red cell lineage in the BM was similar among the two anemic groups after adjusting for $P f$ infection, suggesting that the increased prevalence of $P f$ infection in the HIV-uninfected group could induce an increased red cell production in the BM-which has already been suggestedconfounding the difference observed in the unadjusted analysis ${ }^{21}$ (Achtman et al., manuscript in preparation).

Unlike previous observations, in this study, we did not observe a difference in ID prevalence, defined by the BM iron content between anemic HIV-infected and anemic HIVuninfected groups. ${ }^{6}$ This discrepancy may be explained by the use or not of ferritin levels in the definitions of ID. ${ }^{22,23}$ Serum ferritin is an acute-phase protein that rises during inflammation, and its level is frequently increased in HIV patients, not reflecting iron stores. ${ }^{19,22,23}$ Besides BM content, we determined iron status using an ID definition based on the TfR-F index adjusted by the CRP. The effect modifier of HIV in the risk of anemia due to ID based on the TfR-F index was borderline significant, suggesting that some of the parameters used in this index may be affected by the inflammation associated with HIV infection. The results also show that unlike in uninfected children, there is no difference in the risk of anemia among HIV-infected children with or without ID. This absence of difference might be explained by other more important factors contributing to anemia among HIV-infected children (such as albumin deficiency). Importantly, the prevalence of ID-defined by the BM iron content-was common among HIV-infected children (78\%), with prevalence similar to that in uninfected children (80\%). In settings with high-infectious pressure, iron supplementation to treat and prevent ID is controversial because of reports on the association of high iron levels with worse clinical prognoses, and of iron supplementation and increased risk of malaria episodes. ${ }^{8,24}$ The WHO has published new guidelines on ID prevention in malaria-endemic areas recommending universal iron supplementation; however, these guidelines do not contain specific recommendations for HIV-infected individuals. ${ }^{25}$ Given the high prevalence of ID observed, and given that iron supplementation has shown to improve $\mathrm{Hb}$ levels, reduce anemia prevalence, and increase CD4 percentage, iron provision to HIV-infected children should be considered to prevent anemia in this vulnerable population. ${ }^{8}$

In this study, HIV-infected children had a reduced risk of $P f$ infection and clinical malaria and lower prevalence of $P f$ parasites, gametocytes, and hemozoin in BM. Unlike adults, in whom HIV infection is a risk factor for severe malaria, in children, the effect of HIV on malaria risk is less clear, possibly 
TABLE 4

Association between clinical variables related to anemia and anemia adjusted for HIV and effect of modifier (interaction) of the HIV infection

\begin{tabular}{|c|c|c|c|c|}
\hline Variable & No of observations ${ }^{\star}$ & Odds ratio of anemia & $(95 \% \mathrm{Cl})$ & $P$-value \\
\hline Iron deficiency by TfR-F index by C-reactive protein & 580 & 3.28 & $(2.27 ; 4.73)$ & $<0.0001$ \\
\hline Effect modifier & & 0.06 & $(0.00 ; 1.25)$ & 0.0696 \\
\hline Parvovirus B19 infection & 662 & 4.07 & $(1.58 ; 10.46)$ & 0.0036 \\
\hline Effect modifier & & 0.09 & $(0.00 ; 2.31)$ & 0.1447 \\
\hline EBV positive viral load & 660 & 1.64 & $(1.05 ; 2.57)$ & 0.0301 \\
\hline Effect modifier & & 6.53 & $(0.35 ; 123.51)$ & 0.2107 \\
\hline Acute EBV infection & 633 & 2.47 & $(0.91 ; 6.69)$ & 0.0752 \\
\hline Effect modifier & & 0.58 & $(0.03 ; 13.14)$ & 0.7303 \\
\hline EBV reactivation & 633 & 1.36 & $(0.79 ; 2.35)$ & 0.2665 \\
\hline Effect modifier & & 4.25 & $(0.22 ; 82.30)$ & 0.3384 \\
\hline Wasting (WHZ <-2SD) & 651 & 4.05 & $(2.32 ; 7.07)$ & $<0.0001$ \\
\hline Effect modifier & & 1.36 & $(0.20 ; 9.43)$ & 0.7531 \\
\hline Stunting (HAZ < -2SD) & 654 & 2.37 & $(1.52 ; 3.69)$ & 0.0001 \\
\hline Effect modifier & & 6.94 & $(0.37 ; 131.02)$ & 0.1964 \\
\hline Underweight (WAZ < -2SD) & 659 & 4.96 & $(3.17 ; 7.77)$ & $<0.0001$ \\
\hline Effect modifier & & 1.88 & $(0.28 ; 12.76)$ & 0.5169 \\
\hline Severe wasting (WHZ <-3SD) & 651 & 3.18 & $(1.45 ; 6.96)$ & 0.0039 \\
\hline Effect modifier & & 2.97 & $(0.15 ; 60.16)$ & 0.4789 \\
\hline Severe stunting (HAZ <-3SD) & 654 & 1.42 & $(0.69 ; 2.96)$ & 0.3433 \\
\hline Effect modifier & & 5.54 & $(0.28 ; 110.96)$ & 0.2628 \\
\hline Severe underweight (WAZ <-3SD) & 659 & 4.71 & $(2.49 ; 8.91)$ & $<0.0001$ \\
\hline Effect modifier & & 1.03 & $(0.15 ; 7.29)$ & 0.9772 \\
\hline Albumin deficiency & 647 & 13.26 & $(6.84 ; 25.71)$ & $<0.0001$ \\
\hline Effect modifier & & 1.29 & $(0.06 ; 25.84)$ & 0.8694 \\
\hline Prealbumin deficiency & 643 & 18.19 & $(11.39 ; 29.05)$ & $<0.0001$ \\
\hline Effect modifier & & 0.63 & $(0.10 ; 3.87)$ & 0.6160 \\
\hline Vitamin A deficiency & 650 & 5.84 & $(4.05 ; 8.42)$ & $<0.0001$ \\
\hline Effect modifier & & 0.48 & $(0.09 ; 2.70)$ & 0.4079 \\
\hline Vitamin B12 deficiency & 617 & 0.85 & $(0.55 ; 1.32)$ & 0.4767 \\
\hline Effect modifier & & 0.58 & $(0.08 ; 4.28)$ & 0.5943 \\
\hline Bacteremia & 650 & 4.83 & $(1.52 ; 15.42)$ & 0.0078 \\
\hline Effect modifier & & 0.49 & $(0.02 ; 11.47)$ & 0.6578 \\
\hline Pf. infection & 648 & 11.67 & $(6.97 ; 19.55)$ & $<0.0001$ \\
\hline Effect modifier & & 0.30 & $(0.02 ; 5.89)$ & 0.4308 \\
\hline Hyperparasitemic Pf & 648 & 56.48 & $(3.43 ; 930.83)$ & 0.0048 \\
\hline Effect modifier & & 0.015 & $(0.00 ; 0.90)$ & 0.0444 \\
\hline Submicroscopic $P f$ & 556 & 3.12 & $(1.52 ; 6.42)$ & 0.0020 \\
\hline Effect modifier & & 0.60 & $(0.03 ; 12.39)$ & 0.7378 \\
\hline Hookworm infection & 332 & 0.45 & $(0.15 ; 1.40)$ & 0.1682 \\
\hline Effect modifier & & 0.33 & $(0.01 ; 12.33)$ & 0.5474 \\
\hline
\end{tabular}

because of their immunologically naive status regarding $P f$ parasites, and is less studied. ${ }^{7}$ Previous studies reported a lower risk of $P f$ infection in HIV-infected children. ${ }^{26,27}$ However, in other studies, no differences in the prevalence of parasitemia by HIV status were reported, although the increasing frequency of clinical malaria with the severity of immunosuppression was observed. ${ }^{28-31}$ One explanation for these discrepancies between cross-sectional studies in admitted patients and cohort studies of HIV-infected patients might be that HIV-infected children consult more frequently for other pathologies related to HIV infection, such as pneumonia, diarrhea, or bacteremia, and less for malaria (competing risk theory). ${ }^{26-30,32,33}$ This hypothesis is supported by the observation that the prevalence of hemozoin or gametocytes in the $\mathrm{BM}$ among children without $P f$ peripheral parasitemia-a sign of past malaria infection-was similar between HIV-infected and uninfected children. ${ }^{34}$ The antimalarial effect of cotrimoxazole prophylaxis may also be associated with reduced risk of malaria in the HIV-infected group, ${ }^{35}$ although the adherence to this prophylaxis was very poor in the study population, and less than half of the HIV-infected patients knew their status before the recruitment into the study (Table 1). A limitation of the study is that the information of the children who were on cotrimoxazole prophylaxis relied on the response of the caregivers; in addition, this information was not available in $62 \%$ of the cases. On the other hand, it is also possible that some infants not infected but HIV-exposed could be on cotrimoxazole prophylaxis in the HIV-uninfected group. Importantly, HIV-infected children had a lower risk of having anemia related to $P f$ hyperparasitemia than HIVuninfected children. This may be explained by the fact that in HIV-infected patients, anemia due to other etiological factors is already present before malaria infection occurs, decreasing the relative contribution of malaria hyperparasitemia as a cause of anemia in this group.

Unlike HIV-infected adults, in whom EPO levels do not correlate with $\mathrm{Hb}$ levels, ${ }^{36}$ we found that EPO production was similar in HIV-infected and uninfected children. Moreover, as reported previously in African children, the proportion of HIVinfected children with elevated EPO levels was higher than 
that of uninfected children. ${ }^{23}$ Further studies are needed to determine whether the presence of anti-EPO antibodies or an altered EPO responsiveness might play a role in the pathophysiology of HIV-related anemia. The prevalence of hemolysis was lower in HIV-infected children than that in the uninfected group even after adjusting for Pf infection, suggesting that the pathophysiology of anemia in HIV infection does not have a relevant hemolytic component.

Protein-caloric malnutrition has been described as an etiological factor for anemia, although the pathophysiologic mechanisms are not well understood. ${ }^{14,37}$ The adverse effects of HIV infection on nutritional status have been widely reported, which agrees with the increased risk of malnutrition among HIV-infected children in this study. ${ }^{12}$ Nutritional support to HIV-infected patients to reduce malnutrition-related anemia must be a priority because of its high prevalence, although HIV infection did not increase the risk of anemia related to malnutrition.

Parvovirus infection may cause chronic anemia, and antibodies against this virus are reported to be more prevalent in HIV-infected individuals than in HIV-uninfected populations. ${ }^{38}$ As in other reports, in this study, no differences were observed in parvovirus infection prevalence between HIV-infected and uninfected children. ${ }^{39}$ Epstein-Barr viral load positive and reactivation were more frequent among HIV-infected children; however, the risk of anemia associated with EBV infection did not change by HIV status. Furthermore, EBV infection was not independently associated with anemia, indicating that its prevention might not have an impact on anemia prevalence. ${ }^{14}$

The frequency of bacteremia is increased among HIVinfected patients, which may be a cause of anemia and a mediating factor of the effect of HIV on severe anemia. ${ }^{14} \mathrm{Ac}$ cordingly, in this study, bacteremia was more frequent in HIVinfected children than in uninfected children. However, HIV infection did not modify the risk of anemia secondary to bacteremia. The increased prevalence of bacteremia among admitted HIV-infected children would justify the empirical administration of antibiotics in the management of anemia in settings where blood cultures are not available.

No information is reported on the association of helminthic infections, commonly associated with anemia, and HIV infection in children. In this study, although numbers were small, no differences in the prevalence of hookworm infection between the two groups of children were observed.

The immune status of the HIV-infected children may influence the findings in the BM; as most of the patients were in the early stages of the disease, the BM could be less affected, and differences among groups are less marked. Results of CD4 counts were not available because of limited access to this analysis at the time of the study, and the clinical stage of the children was not recorded, which is a limitation of the study. However, nearly $70 \%$ of the HIV-infected children had high viral loads, suggesting they were in advanced disease. Although ART could affect the BM response-improving disease and HIV-related anemia or affecting erythropoiesis due to drug toxicity-only $10 \%$ of the children were on ART, so the influence of this factor in the HIV-infected children is expected to be limited in both ways. Another study limitation is that some parents refused the HIV test for their children, introducing a potential bias because these are likely to have been infected. It is also important to notice that the observed associations among the study variables and anemia among
HIV-infected children cannot be considered as the cause of their anemia because of the case-control design of the study. However, all study variables are known causes of anemia and should be taken into account as a possible relevant cause of anemia in this population, mainly those that have been associated with HIV infection in this study..$^{37,40-44}$ On the other hand, inflammation has not been studied as an independent etiology of anemia in this study, as it is very unspecific. Creactive protein was higher in the anemic and the HIV-infected group (Table 1); however, the cause of the observed high CRP levels could be related to several infections limiting the public health implications of this observation. Therefore, the role of anemia of inflammation in the physiopathology of anemia in HIV-infected children should be considered. The potential bias caused by rare events due to the small number of HIVinfected controls had been mitigated by using the penalized likelihood for estimating logistic regression models. ${ }^{18}$ Finally, although this analysis is based on information collected several years ago, unfortunately, these findings are still relevant to guide policies to reduce the burden of anemia in HIV-infected children exposed to malaria, given the scarcity of interventions focused on reducing this problem in this vulnerable group.

In summary, the pathophysiology of anemia among HIVinfected malaria-exposed children does not seem to be associated with a specific effect of the HIV infection. Nevertheless, other common causes of anemia, such as undernutrition or bacteremia, may play a role in the anemia etiology in HIV-infected children because of the high prevalence of these conditions. Iron deficiency prevalence was comparable in HIV-infected and uninfected children, suggesting that iron supplementation recommendations should not be different in HIV-infected children.

Received October 23, 2019. Accepted for publication September 24, 2020.

Note: Supplemental tables appear at www.ajtmh.org.

Acknowledgments: We are grateful to the parents and their children participating in the study. We are also grateful to the dedicated staff of the Manhiça District Hospital, as well as the laboratory, clinical, field, and data management staff at the Centro de Investigaçaõ em Saúde de Manhiça, Mozambique, and to the investigators from the Hospital Clínic de Barcelona who performed laboratory measurements. ISGlobal is a member of the CERCA Programme, Generalitat de Catalunya.

Financial support: This study was funded by the Agencia de Cooperación Internacional de Las Illes Balears, Spain, and the Fundación Ramón Areces. The Centro de Investigaçaõ em Saúde de Manhiça receives core funding from the Spanish Agency for International Cooperation and Development (AECID). The Walter and Eliza Hall Institute receives Victorian State Government Operational Infrastructure Support and Australian Government NHMRC IRIISS. L. S. received support as an International Research Scholars of the Howard Hughes Medical Institute and from NHMRC program grant \#637406. AHA received support from the Victorian Community Foundation-the George Perry Fund.

Authors' addresses: Cinta Moraleda, ISGlobal, Hospital Clínic - Universitat de Barcelona, Barcelona, Spain, and Pediatric Infectious Diseases Unit, Instituto de investigación Hospital, RiTiP, Hospital Universitario, Universidad Complutense, Madrid, Spain, E-mail: cinta.moraleda@isglobal.org. Ruth Aguilar, Llorenç Quintó, Montserrat Renom, John J. Aponte, and Clara Menéndez, ISGlobal, Hospital Clínic Universitat de Barcelona, Barcelona, Spain, E-mails: ruth.aguilar@ isglobal.org, llorenc.quinto@isglobal.org, montse.renom034@gmail. com, john.aponte@isglobal.org, and clara.menendez@isglobal.org. Tacilta Nhampossa, Augusto Nhabomba, and Eusebio Macete, Manhiça Health Research Center (CISM), Manhiça, Mozambique, 
E-mails: tacilta.nhampossa@manhica.net, augusto.nhabomba@ gmail.com, and eusebio.macete@manhica.net. María Ruperez, London School of Hygiene and Tropical Medicine, London, United Kingdom, E-mail: maria.ruperez@Ishtm.ac.uk. Ariel H. Achtman and Louis Schofield, Walter and Eliza Hall Institute for Medical Research, Parkville, Australia, E-mails: arielachtman@hotmail.com and louis. schofield@jcu.edu.au. María del Mar Mañú Pereira, Red Blood Cell Pathology- Centre for Rare Diseases, Vall d'Hebron Research Institute, University Hospital Vall d'Hebron, Barcelona, Spain, E-mail: mar.manu@vhir.org. Pedro L. Alonso, ISGlobal, Hospital Clínic Universitat de Barcelona, Barcelona, Spain, and Global Malaria Programme, WHO, Geneva, Switzerland, Email: alonsop@who.int.

\section{REFERENCES}

1. Calis JC, van Hensbroek MB, de Haan RJ, Moons P, Brabin BJ, Bates I, 2008. HIV-associated anemia in children: a systematic review from a global perspective. AIDS 22: 1099-1112.

2. Aguilar R, Moraleda C, Quinto L, Renom M, Mussacate L, Macete E, Aguilar JL, Alonso PL, Menendez C, 2012. Challenges in the diagnosis of iron deficiency in children exposed to high prevalence of infections. PLoS One 7: e50584.

3. Mir N, Costello C, Luckit J, Lindley R, 1989. HIV-disease and bone marrow changes: a study of 60 cases. Eur J Haematol 42: 339-343.

4. Mueller BU, Tannenbaum S, Pizzo PA, 1996. Bone marrow aspirates and biopsies in children with human immunodeficiency virus infection. J Pediatr Hematol Oncol 18: 266-271.

5. Meira DG, Lorand-Metze I, Toro AD, Silva MT, Vilela MM, 2005. Bone marrow features in children with HIV infection and peripheral blood cytopenias. J Trop Pediatr 51: 114-119.

6. Esan MO, Jonker FA, Hensbroek MB, Calis JC, Phiri KS, 2012. Iron deficiency in children with HIV-associated anaemia: a systematic review and meta-analysis. Trans $R$ Soc Trop Med Hyg 106: $579-587$.

7. Gonzalez R, Ataide R, Naniche D, Menendez C, Mayor A, 2012. HIV and malaria interactions: where do we stand? Expert Rev Anti Infect Ther 10: 153-165.

8. Esan MO, van Hensbroek MB, Nkhoma E, Musicha C, White SA, Ter Kuile FO, Phiri KS, 2013. Iron supplementation in HIVinfected Malawian children with anemia: a double-blind, randomized, controlled trial. Clin Infect Dis 57: 1626-1634.

9. Sacoor $C$ et al., 2013. Profile: manhica health research centre (manhica HDSS). Int J Epidemiol 42: 1309-1318.

10. Gonzalez R, Munguambe K, Aponte J, Bavo C, Nhalungo D, Macete E, Alonso P, Menendez C, Naniche D, 2012. High HIV prevalence in a southern semi-rural area of Mozambique: a community-based survey. HIV Med 13: 581-588.

11. Moraleda C, de Deus N, Serna-Bolea C, Renom M, Quinto L, Macete E, Menendez C, Naniche D, 2014. Impact of HIV exposure on health outcomes in HIV-negative infants born to HIVpositive mothers in sub-Saharan Africa. J Acquir Immune Defic Syndr 65: 182-189.

12. Pedrini M, Moraleda C, Macete E, Gondo K, Brabin BJ, Menendez C, 2015. Clinical, nutritional and immunological characteristics of HIV-infected children in an area of high HIV prevalence. J Trop Pediatr 61: 286-294.

13. WHO, 2006. WHO 2006 Guidelines on ART in Infants and Children in Resource Limited Setting: Towards Universal Access, Recommendations for a Public Health Approach. Geneva, Switzerland: World Health Organization.

14. Moraleda $C$ et al., 2017. Anaemia in hospitalised preschool children from a rural area in Mozambique: a case control study in search for aetiological agents. BMC Pediatr 17: 63.

15. Stoltzfus RJ, Dreyfuss ML, 1998. Guidelines for the Use of Iron Supplements to Prevent and Treat Iron Deficiency Anemia. Washington, DC: International Nutritional Anemia Consultative Group (INACG), World Health Organization (WHO), United Nations Childrens Fund (UNICEF).

16. Sapero JJ, Lawless DK, 1953. The MIF stain-preservation technic for the identification of intestinal protozoa. Am J Trop Med Hyg 2: 613-619.

17. Romagosa C, Menendez C, Ismail MR, Quinto L, Ferrer B, Alonso PL, Ordi J, 2004. Polarisation microscopy increases the sensitivity of hemozoin and Plasmodium detection in the histological assessment of placental malaria. Acta Trop 90: 277-284.

18. Heinze G, Schemper M, 2002. A solution to the problem of separation in logistic regression. Stat Med 21: 2409-2419.

19. Salome MA, Grotto HZ, 2002. Human immunodeficiency virusrelated anemia of chronic disease: relationship to hematologic, immune, and iron metabolism parameters, and lack of association with serum interferon-gamma levels. AIDS Patient Care STDS 16: 361-365.

20. Calis JC et al., 2010. Erythropoiesis in HIV-infected and uninfected Malawian children with severe anemia. AIDS 24: 2883-2887.

21. Menendez C, Quinto LL, Kahigwa E, Alvarez L, Fernandez R, Gimenez N, Schellenberg D, Aponte JJ, Tanner M, Alonso PL, 2001. Effect of malaria on soluble transferrin receptor levels in Tanzanian infants. Am J Trop Med Hyg 65: 138-142.

22. Totin D, Ndugwa C, Mmiro F, Perry RT, Jackson JB, Semba RD, 2002. Iron deficiency anemia is highly prevalent among human immunodeficiency virus-infected and uninfected infants in Uganda. J Nutr 132: 423-429.

23. Miller MF, Humphrey JH, Iliff PJ, Malaba LC, Mbuya NV, Stoltzfus RJ; ZVITAMBO Study Group, 2006. Neonatal erythropoiesis and subsequent anemia in HIV-positive and HIV-negative Zimbabwean babies during the first year of life: a longitudinal study. BMC Infect Dis 6: 1.

24. Gordeuk VR, Delanghe JR, Langlois MR, Boelaert JR, 2001. Iron status and the outcome of HIV infection: an overview. J Clin Virol 20: 111-115.

25. WHO, 2016. Guideline: Daily Iron Supplementation in Infants and Children. Geneva, Switzerland: World Health Organization.

26. Bebell LM, Gasasira A, Kiggundu M, Dokomajilar C, Kamya MR, Charlebois ED, Havlir D, Rosenthal PJ, Dorsey G, 2007. HIV-1 infection in patients referred for malaria blood smears at government health clinics in Uganda. J Acquir Immune Defic Syndr 46: 624-630.

27. Villamor E, Fataki MR, Mbise RL, Fawzi WW, 2003. Malaria parasitaemia in relation to HIV status and vitamin A supplementation among pre-school children. Trop Med Int Health 8: 1051-1061.

28. Greenberg AE, Nsa W, Ryder RW, Medi M, Nzeza M, Kitadi N, Baangi M, Malanda N, Davachi F, Hassig SE, 1991. Plasmodium falciparum malaria and perinatally acquired human immunodeficiency virus type 1 infection in Kinshasa, Zaire. A prospective, longitudinal cohort study of 587 children. N Engl J Med 325: 105-109.

29. Kalyesubula I, Musoke-Mudido P, Marum L, Bagenda D, Aceng E, Ndugwa C, Olness K, 1997. Effects of malaria infection in human immunodeficiency virus type 1-infected Ugandan children. Pediatr Infect Dis J 16: 876-881.

30. Colebunders R et al., 1990. Incidence of malaria and efficacy of oral quinine in patients recently infected with human immunodeficiency virus in Kinshasa, Zaire. J Infect 21: 167-173.

31. Flateau C, Le Loup G, Pialoux G, 2011. Consequences of HIV infection on malaria and therapeutic implications: a systematic review. Lancet Infect Dis 11: 541-556.

32. Meyers TM, Pettifor JM, Gray GE, Crewe-Brown H, Galpin JS, 2000. Pediatric admissions with human immunodeficiency virus infection at a regional hospital in Soweto, South Africa. $J$ Trop Pediatr 46: 224-230.

33. Dignam JJ, Zhang Q, Kocherginsky M, 2012. The use and interpretation of competing risks regression models. Clin Cancer Res 18: 2301-2308.

34. Ismail MR, Ordi J, Menendez C, Ventura PJ, Aponte JJ, Kahigwa E, Hirt R, Cardesa A, Alonso PL, 2000. Placental pathology in malaria: a histological, immunohistochemical, and quantitative study. Hum Pathol 31: 85-93.

35. Dow A et al., 2012. Effects of cotrimoxazole prophylactic treatment on adverse health outcomes among HIV-exposed, uninfected infants. Pediatr Infect Dis J 31: 842-847.

36. Spivak JL, Barnes DC, Fuchs E, Quinn TC, 1989. Serum immunoreactive erythropoietin in HIV-infected patients. JAMA. 261: 3104-3107. 
37. Macdougall LG, Moodley G, Eyberg C, Quirk M, 1982. Mechanisms of anemia in protein-energy malnutrition in Johannesburg. Am J Clin Nutr 35: 229-235.

38. Vernazza PL, Pfister LA, Siegl G, Cassinotti P, 1996. High seroprevalence of parvovirus B19 among patients infected with human immunodeficiency virus. Clin Infect Dis 22: 198-199.

39. LaMonte AC, Paul ME, Read JS, Frederick MM, Erdman DD, Han LL, Anderson LJ, 2004. Persistent parvovirus B19 infection without the development of chronic anemia in HIV-infected and -uninfected children: the women and infants transmission study. J Infect Dis 189: 847-851.

40. Menendez C, Fleming AF, Alonso PL, 2000. Malaria-related anaemia. Parasitol Today 16: 469-476.
41. Morinet F, Leruez-Ville M, Pillet S, Fichelson S, 2011. Concise review: anemia caused by viruses. Stem Cells 29: 16561660.

42. Calis JC et al., 2008. Severe anemia in Malawian children. NEngl J Med 358: 888-899.

43. Watkins D, Whitehead VM, Rosenblatt DS, 2009. Megaloblastic Anemia. Nathan and Oski's Hematology of Infancy and Childhood. Philadelphia, PA: Saunders Elsevier, 469-501.

44. Graham SM, Walsh AL, Molyneux EM, Phiri AJ, Molyneux ME, 2000. Clinical presentation of non-typhoidal Salmonella bacteraemia in Malawian children. Trans $R$ Soc Trop Med Hyg 94: 310-314. 